International Journal of Pure and Applied Mathematics

Volume 107 No. 4 2016, 845-858

ISSN: 1311-8080 (printed version); ISSN: 1314-3395 (on-line version)

url: http://www.ijpam.eu

doi: 10.12732/ijpam.v107i4.4

\title{
CONSTRAINED LINEARLY ELASTIC MATERIALS REINFORCED BY TWO FAMILIES OF FIBRES
}

\author{
Maria Luisa Tonon \\ Department of Mathematics "Giuseppe Peano" \\ University of Turin \\ Via Carlo Alberto 10, Turin, 10123, ITALY
}

\begin{abstract}
In this paper the linearized finite theory of elasticity is applied to obtain linear constitutive equations for constrained materials reinforced by two orhogonal families of fibres. Explicit expressions for the three stress tensors are derived for inextensible in both fibre directions materials, for incompressible materials and finally for materials that are both incompressible and inextensible in the two fibre directions. Comparison with the corresponding constitutive equations provided by the classical approach shows that the classical equations are not accurate to first order in the strain.
\end{abstract}

AMS Subject Classification: 74B99, 74E10, 74A10

Key Words: constraints, linearized finite elasticity, constitutive equations, orthotropic symmetry

\section{Introduction}

The aim of this paper is to apply the linearized finite theory of elasticity to obtain the constitutive equations for constrained hyperelastic materials reinforced by two families of fibres.

The linearized finite theory of elasticity (LFTE in the following) formulated by Hoger and Johnson in [1], [2] and Marlow in [3] provides linear constitutive equations for constrained materials derived by linearization of the corresponding finite constitutive equations. Recent contributions concerning the constitutive

Received: March 17, 2016

Published: May 4, 2016 (c) 2016 Academic Publications, Ltd.

url: www.acadpubl.eu 
equations for the three stress tensors according to LFTE can be found in [10], while in [11] the method of LFTE is extended to second-order constitutive equations. The results obtained in [1], [2], [3], [10] emphasize the need to use the LFTE in order to have the accuracy required by a linear model, since the classical linear theory of elasticity (CLTE in the following) provides constitutive equations that are not accurate to first order in the displacement gradient. The application of LFTE also to static problems (see [1], [3], [8], [9]) or dynamical problems (see [6], [7]) confirms the inadequacy of CLTE.

In this paper, starting from [2], [10], in Section 3 we obtain the constitutive equations for the three stress tensors appropriate for constrained materials with orthotropic symmetry according to LFTE. In Section 4 we provide explicit results for fibre-reinforced materials inextensible in both fibre directions, for incompressible materials and for fibre-reinforced incompressible materials inextensible in both fibre directions. Finally in Section 5 we compare our constitutive equations with the constitutive equations obtained by Spencer in [4], [5] according to the classical approach. Comparison shows that also for orthotropic constrained materials the stress-strain relations provided by CLTE are not accurate to first-order in the strain.

\section{Linear Constitutive Equations According to the Linearized Finite Theory of Elasticity}

In this section we briefly recall the constitutive equations appropriate for the so-called linearized finite theory of elasticity for solid hyperelastic constrained materials. We refer to [2], [10] for all details concerning the procedure of linearization.

Denote by $\mathcal{B}_{0}$ and $\mathcal{B}=\mathbf{f}\left(\mathcal{B}_{0}\right)$ the reference configuration and the deformed configuration, respectively; $\mathbf{f}$ is the deformation function which carries the point $\mathbf{X} \in \mathcal{B}_{0}$ into the point $\mathbf{x}=\mathbf{f}(\mathbf{X}) \in \mathcal{B}$.

The displacement $\mathbf{u}$, the deformation gradient $\mathbf{F}$, the displacement gradient $\mathbf{H}$, the right Cauchy-Green deformation tensor $\mathbf{C}$ and the finite Green strain tensor $\mathbf{E}_{G}$ are defined as follows

$$
\begin{aligned}
\mathbf{u}(\mathbf{X}) & =\mathbf{f}(\mathbf{X})-\mathbf{X}, \\
\mathbf{F} & =\operatorname{Grad} \mathbf{f}, \\
\mathbf{H} & =\operatorname{Grad} \mathbf{u}=\mathbf{F}-\mathbf{I}, \\
\mathbf{C} & =\mathbf{F}^{T} \mathbf{F},
\end{aligned}
$$




$$
\mathbf{E}_{G}=\frac{1}{2}(\mathbf{C}-\mathbf{I})
$$

respectively; in the previous formulas Grad denotes the gradient operator with respect to $\mathbf{X}$, while $\mathbf{I}$ is the identity tensor.

For an elastic material subject to a single constraint the finite constraint equation is

$$
\hat{c}\left(\mathbf{E}_{G}\right)=0
$$

for a constrained finite hyperelastic material with strain energy density $W=$ $\hat{W}\left(\mathbf{E}_{G}\right)$ the Cauchy stress $\mathbf{T}$ is given by

$$
\mathbf{T}=\mathbf{T}_{d}+\mathbf{T}_{r}
$$

where $\mathbf{T}_{d}$ and $\mathbf{T}_{r}$ are the determinate stress and the reaction stress, defined as follows

$$
\begin{aligned}
& \mathbf{T}_{d}=(\operatorname{det} \mathbf{F})^{-1} \mathbf{F} \frac{\partial \hat{W}}{\partial \mathbf{E}_{G}}\left(\mathbf{E}_{G}\right) \mathbf{F}^{T} \\
& \mathbf{T}_{r}=q \mathbf{F} \frac{\partial \hat{c}}{\partial \mathbf{E}_{G}}\left(\mathbf{E}_{G}\right) \mathbf{F}^{T}
\end{aligned}
$$

respectively.

Note that in (8) the strain energy density for the constrained material is taken to be that of the unconstrained material with the same material symmetry; moreover in (9) $q$ denotes a Lagrange multiplier.

Finally we recall that in finite elasticity the first Piola-Kirchhoff stress $\mathbf{S}$ and the second Piola-Kirchhoff stress $\tilde{\mathbf{T}}$ are given in terms of the Cauchy stress $\mathbf{T}$ by the following relations

$$
\begin{aligned}
\mathbf{S} & =(\operatorname{det} \mathbf{F}) \mathbf{T} \mathbf{F}^{-T}, \\
\tilde{\mathbf{T}} & =(\operatorname{det} \mathbf{F}) \mathbf{F}^{-1} \mathbf{T} \mathbf{F}^{-T},
\end{aligned}
$$

respectively.

In order to linearize equations (8), (9), (10), (11) about the zero strain state according to LFTE, we require $\|\mathbf{H}\| \rightarrow 0$ and we adopt a procedure of linearization in which the linearization of the derivative of $\hat{W}$ parallels that of the derivative of $\hat{c}$. Moreover, according to a linear theory, we use the following expansions

$$
\begin{aligned}
& \operatorname{det} \mathbf{F} \cong 1+\operatorname{tr} \mathbf{H}, \\
&(\operatorname{det} \mathbf{F})^{-1} \cong 1-\operatorname{tr} \mathbf{H},
\end{aligned}
$$




$$
\begin{aligned}
\mathbf{F}^{-1} & \cong \mathbf{I}-\mathbf{H} \\
\mathbf{F}^{-T} & \cong \mathbf{I}-\mathbf{H}^{T}, \\
\mathbf{E}_{G} & \cong \mathbf{O}+\frac{1}{2}\left(\mathbf{H}+\mathbf{H}^{T}\right), \\
\hat{c}\left(\mathbf{E}_{G}\right) & \cong \frac{1}{2} \frac{\partial \hat{c}}{\partial \mathbf{E}_{G}}(\mathbf{O}) \cdot\left(\mathbf{H}+\mathbf{H}^{T}\right), \\
\frac{\partial \hat{W}}{\partial \mathbf{E}_{G}}\left(\mathbf{E}_{G}\right) & \cong \frac{1}{2} \frac{\partial^{2} \hat{W}}{\partial \mathbf{E}_{G} \partial \mathbf{E}_{G}}(\mathbf{O})\left(\mathbf{H}+\mathbf{H}^{T}\right), \\
\frac{\partial \hat{c}}{\partial \mathbf{E}_{G}}\left(\mathbf{E}_{G}\right) & \cong \frac{\partial \hat{c}}{\partial \mathbf{E}_{G}}(\mathbf{O})+\frac{1}{2} \frac{\partial^{2} \hat{c}}{\partial \mathbf{E}_{G} \partial \mathbf{E}_{G}}(\mathbf{O})\left(\mathbf{H}+\mathbf{H}^{T}\right) .
\end{aligned}
$$

In the previous formulas $\mathbf{O}$ is the zero tensor, while the symbol d denotes scalar product. Expansion (17) takes into account the condition $\hat{c}(\mathbf{O})=0$ provided by (6), while expansion (18) has been obtained under the hypothesis of zero residual stress, that is $\frac{\partial \hat{W}}{\partial \mathbf{E}_{G}}(\mathbf{O})=\mathbf{O}$.

The final expressions for the three stress tensors appropriate for LFTE are

$$
\begin{aligned}
\mathbf{T} \cong & \left.\frac{1}{2} \frac{\partial^{2} \hat{W}}{\partial \mathbf{E}_{G} \partial \mathbf{E}_{G}}(\mathbf{O})\right|_{\tilde{c}}\left(\mathbf{H}+\mathbf{H}^{T}\right)+q\left\{\frac{\partial \hat{c}}{\partial \mathbf{E}_{G}}(\mathbf{O})+\mathbf{H} \frac{\partial \hat{c}}{\partial \mathbf{E}_{G}}(\mathbf{O})\right. \\
& \left.+\frac{\partial \hat{c}}{\partial \mathbf{E}_{G}}(\mathbf{O}) \mathbf{H}^{T}+\frac{1}{2} \frac{\partial^{2} \hat{c}}{\partial \mathbf{E}_{G} \partial \mathbf{E}_{G}}(\mathbf{O})\left(\mathbf{H}+\mathbf{H}^{T}\right)\right\} \\
\mathbf{S} \cong & \left.\frac{1}{2} \frac{\partial^{2} \hat{W}}{\partial \mathbf{E}_{G} \partial \mathbf{E}_{G}}(\mathbf{O})\right|_{\tilde{c}}\left(\mathbf{H}+\mathbf{H}^{T}\right)+q\left\{(1+\operatorname{tr} \mathbf{H}) \frac{\partial \hat{c}}{\partial \mathbf{E}_{G}}(\mathbf{O})\right. \\
& \left.+\mathbf{H} \frac{\partial \hat{c}}{\partial \mathbf{E}_{G}}(\mathbf{O})+\frac{1}{2} \frac{\partial^{2} \hat{c}}{\partial \mathbf{E}_{G} \partial \mathbf{E}_{G}}(\mathbf{O})\left(\mathbf{H}+\mathbf{H}^{T}\right)\right\} \\
\tilde{\mathbf{T}} \cong & \left.\frac{1}{2} \frac{\partial^{2} \hat{W}}{\partial \mathbf{E}_{G} \partial \mathbf{E}_{G}}(\mathbf{O})\right|_{\tilde{c}}\left(\mathbf{H}+\mathbf{H}^{T}\right)+q\left\{(1+\operatorname{tr} \mathbf{H}) \frac{\partial \hat{c}}{\partial \mathbf{E}_{G}}(\mathbf{O})\right. \\
& \left.+\frac{1}{2} \frac{\partial^{2} \hat{c}}{\partial \mathbf{E}_{G} \partial \mathbf{E}_{G}}(\mathbf{O})\left(\mathbf{H}+\mathbf{H}^{T}\right)\right\}
\end{aligned}
$$

(see [2], formulas (3.22), (3.23), and [10], formula (34)).

In $(20),(21),(22)$ the subscript $\tilde{c}$ denotes evaluation on the linearized constraint equation

$$
\tilde{c}(\mathbf{E})=0
$$

where

$$
\mathbf{E}=\frac{1}{2}\left(\mathbf{H}+\mathbf{H}^{T}\right)
$$


is the infinitesimal strain tensor and

$$
\tilde{c}(\mathbf{E})=\frac{\partial \hat{c}}{\partial \mathbf{E}_{G}}(\mathbf{O}) \cdot \mathbf{E}
$$

is the linear constraint function.

Note that in LFTE the three stress tensors differ by terms that are first order in $\mathbf{H}$ (the determinate stress is the same, but the reaction stress is different), while in classical linear theory of elasticity the three stress tensors coincide, both for unconstrained and constrained materials.

\section{Linear Constitutive Equations for Constrained Materials Reinforced by two Families of Fibres According to LFTE}

In this section we apply the results exposed in Section 1 to constrained materials which are reinforced by two orthogonal families of fibres. Then the material symmetry appropriate for such bodies is the orthotropic symmetry.

According to LFTE, our procedure of linearization starts from the constitutive equations which describe the behaviour of finite hyperelastic orthotropic materials; we refer to the costitutive equations given by Spencer in [4], Section 6.5, and [5], Section 3.3.

Let $\mathcal{B}$ be a finite elastic body reinforced by two families of fibres, whose directions in the reference configuration are defined by two unit vectors $\mathbf{a}$ and $\mathbf{b}$; if the two families of fibres are orthogonal in the reference configuration, the material is orthotropic in this configuration.

For a finite hyperelastic body the strain energy density $W=\hat{W}\left(\mathbf{E}_{G}\right)$ is a function of the polynomial invariants of the strain appropriate for the material symmetry; denoting by $l\left(\mathbf{E}_{G}\right)$ the complete list of the invariants of $\mathbf{E}_{G}$, we have

$$
\hat{W}\left(\mathbf{E}_{G}\right)=\hat{\omega}\left(l\left(\mathbf{E}_{G}\right)\right) .
$$

Then for a finite hyperelastic body with orthotropic symmetry $W$ can be expressed as a function of the following list of seven invariants

$$
l\left(\mathbf{E}_{G}\right)=\left\{I_{1}, I_{2}, I_{3}, I_{4}, I_{5}, I_{6}, I_{7}\right\},
$$


where

$$
\begin{aligned}
I_{1} & =\mathbf{I} \cdot \mathbf{E}_{G} \\
I_{2} & =\mathbf{I} \cdot \mathbf{E}_{G}^{2} \\
I_{3} & =\mathbf{I} \cdot \mathbf{E}_{G}^{3} \\
I_{4} & =\mathbf{a} \cdot \mathbf{E}_{G} \mathbf{a} \\
I_{5} & =\mathbf{a} \cdot \mathbf{E}_{G}^{2} \mathbf{a} \\
I_{6} & =\mathbf{b} \cdot \mathbf{E}_{G} \mathbf{b} \\
I_{7} & =\mathbf{b} \cdot \mathbf{E}_{G}^{2} \mathbf{b}
\end{aligned}
$$

(see [5], Section 3.3).

If $\mathcal{B}$ is a hyperelastic orthotropic body subject to a constraint (6), also the constraint function $\hat{c}\left(\mathbf{E}_{G}\right)$ is expressed as a function of the list $(27)$ of the polynomial invariants (28), that is

$$
\hat{c}\left(\mathbf{E}_{G}\right)=\hat{\xi}\left(l\left(\mathbf{E}_{G}\right)\right) .
$$

We consider now a linear hyperelastic body reinforced by two orthogonal families of fibres subject to a constraint expressed by (23), according to LFTE. Our aim is to derive for such a body the explicit expressions for the three stress tensors by applying formulas (20), (21), (22).

Since (26), (27), (28) hold, the fourth-order tensor $\frac{\partial^{2} \hat{W}}{\partial \mathbf{E}_{G} \partial \mathbf{E}_{G}}(\mathbf{O})$ appearing in $(20),(21),(22)$ can be written in terms of the seven polynomial invariants of the strain as follows

$$
\begin{aligned}
\frac{\partial^{2} \hat{W}}{\partial \mathbf{E}_{G} \partial \mathbf{E}_{G}}(\mathbf{O}) & =\sum_{p=1}^{7} \sum_{q=1}^{7} \frac{\partial^{2} \hat{\omega}}{\partial I_{p} \partial I_{q}}(l(\mathbf{O})) \frac{\partial I_{p}}{\partial \mathbf{E}_{G}}(\mathbf{O}) \otimes \frac{\partial I_{q}}{\partial \mathbf{E}_{G}}(\mathbf{O}) \\
& +\sum_{p=1}^{7} \frac{\partial \hat{\omega}}{\partial I_{p}}(l(\mathbf{O})) \frac{\partial^{2} I_{p}}{\partial \mathbf{E}_{G} \partial \mathbf{E}_{G}}(\mathbf{O}),
\end{aligned}
$$

where the symbol $\otimes$ denotes tensor product.

The first derivatives and the second derivatives of the seven invariants ap- 
pearing in (30) are given by

$$
\begin{aligned}
\frac{\partial I_{1}}{\partial \mathbf{E}_{G}} & =\mathbf{I} \\
\frac{\partial I_{2}}{\partial \mathbf{E}_{G}} & =2 \mathbf{E}_{G} \\
\frac{\partial I_{3}}{\partial \mathbf{E}_{G}} & =3 \mathbf{E}_{G} \\
\frac{\partial I_{4}}{\partial \mathbf{E}_{G}} & =\mathbf{a} \otimes \mathbf{a} \\
\frac{\partial I_{5}}{\partial \mathbf{E}_{G}} & =\mathbf{a} \otimes\left(\mathbf{E}_{G} \mathbf{a}\right)+\left(\mathbf{E}_{G} \mathbf{a}\right) \otimes \mathbf{a} \\
\frac{\partial I_{6}}{\partial \mathbf{E}_{G}} & =\mathbf{b} \otimes \mathbf{b} \\
\frac{\partial I_{7}}{\partial \mathbf{E}_{G}} & =\mathbf{b} \otimes\left(\mathbf{E}_{G} \mathbf{b}\right)+\left(\mathbf{E}_{G} \mathbf{b}\right) \otimes \mathbf{b}
\end{aligned}
$$

and

$$
\begin{aligned}
\frac{\partial^{2} I_{p}}{\partial\left(\mathbf{E}_{G}\right)_{i j} \partial\left(\mathbf{E}_{G}\right)_{k l}}= & 0 \quad(p=1,4,6) \\
\frac{\partial^{2} I_{2}}{\partial\left(\mathbf{E}_{G}\right)_{i j} \partial\left(\mathbf{E}_{G}\right)_{k l}}= & \delta_{i k} \delta_{j l}+\delta_{i l} \delta_{j k} \\
\frac{\partial^{2} I_{3}}{\partial\left(\mathbf{E}_{G}\right)_{i j} \partial\left(\mathbf{E}_{G}\right)_{k l}}= & \frac{3}{2}\left\{\delta_{i k}\left(\mathbf{E}_{G}\right)_{j l}+\delta_{l j}\left(\mathbf{E}_{G}\right)_{k i}\right. \\
& \left.+\delta_{k j}\left(\mathbf{E}_{G}\right)_{i l}+\delta_{l i}\left(\mathbf{E}_{G}\right)_{k j}\right\} \\
\frac{\partial^{2} I_{5}}{\partial\left(\mathbf{E}_{G}\right)_{i j} \partial\left(\mathbf{E}_{G}\right)_{k l}}= & \frac{1}{2}\left(a_{i} a_{l} \delta_{j k}+a_{i} a_{k} \delta_{j l}\right. \\
\frac{\partial^{2} I_{7}}{\partial\left(\mathbf{E}_{G}\right)_{i j} \partial\left(\mathbf{E}_{G}\right)_{k l}}= & \frac{1}{2}\left(a_{i} b_{l} \delta_{l} \delta_{j k}+a_{j} a_{k} \delta_{l i}\right) \\
& \left.+b_{j} b_{l} b_{k} \delta_{i k}+b_{j l} b_{k} \delta_{l i}\right),
\end{aligned}
$$

respectively.

Substitution of (31), (32) into (30) provides for the components of the tensor 
$\frac{\partial^{2} \hat{W}}{\partial \mathbf{E}_{G} \partial \mathbf{E}_{G}}(\mathbf{O})$ the following expression

$$
\begin{aligned}
& \frac{\partial^{2} \hat{W}}{\partial\left(\mathbf{E}_{G}\right)_{i j} \partial\left(\mathbf{E}_{G}\right)_{k l}}(\mathbf{O})=\alpha_{11} \delta_{i j} \delta_{k l}+\alpha_{44} a_{i} a_{j} a_{k} a_{l} \\
& \quad+\alpha_{66} b_{i} b_{j} b_{k} b_{l}+\alpha_{41}\left(\delta_{i j} a_{k} a_{l}+a_{i} a_{j} \delta_{k l}\right) \\
& \quad+\alpha_{61}\left(\delta_{i j} b_{k} b_{l}+b_{i} b_{j} \delta_{k l}\right)+\alpha_{64}\left(b_{i} b_{j} a_{k} a_{l}+a_{i} a_{j} b_{k} b_{l}\right) \\
& \quad+\alpha_{2}\left(\delta_{i k} \delta_{j l}+\delta_{i l} \delta_{j k}\right)+\frac{1}{2} \alpha_{5}\left(a_{i} a_{l} \delta_{j k}+a_{i} a_{k} \delta_{j l}\right. \\
& \left.\quad+a_{j} a_{l} \delta_{i k}+a_{j} a_{k} \delta_{l i}\right)+\frac{1}{2} \alpha_{7}\left(b_{i} b_{l} \delta_{j k}+b_{i} b_{k} \delta_{j l}\right. \\
& \left.\quad+b_{j} b_{l} \delta_{i k}+b_{j} b_{k} \delta_{l i}\right) .
\end{aligned}
$$

The coefficients $\alpha_{11}, \alpha_{44}, \alpha_{66}, \alpha_{41}, \alpha_{61}, \alpha_{64}$ are defined as follows

$$
\alpha_{p q}=\frac{\partial^{2} \hat{\omega}}{\partial I_{p} \partial I_{q}}(l(\mathbf{O})) \quad(p, q=1,4,6),
$$

while the coefficients $\alpha_{2}, \alpha_{5}, \alpha_{7}$ are given by

$$
\alpha_{p}=\frac{\partial \hat{\omega}}{\partial I_{p}}(l(\mathbf{O})) \quad(p=2,5,7) .
$$

Note that the coefficients $\alpha_{11}$ and $\alpha_{2}$ correspond to the Lamé moduli $\lambda$ and $\mu$, respectively. In order to obtain the final expressions (20), (21), (22) for $\mathbf{T}$, $\mathbf{S}, \tilde{\mathbf{T}}$, respectively, the constraint (6) must be specified. Then we can calculate (33) on the linear constraint equation (23); moreover by (20), (21), (22) we can obtain the reaction parts of the three stress tensors.

In the next section we apply the previous results to hyperelastic fibrereinforced materials subject to particular constraints, as inextensibility in both fibre directions and incompressibility.

\section{Examples}

In this section the general constitutive equations for $\mathbf{T}, \mathbf{S}, \tilde{\mathbf{T}}$ obtained in Section 3 for fibre-reinforced materials are specified for inextensible in both fibre directions materials, for incompressible materials and finally for materials which are both incompressible and inextensible in the two fibre directions.

(i) Fibre-reinforced materials inextensible in both fibre directions In this case the body is subject to two constraints. The finite constraint equations are 


$$
\begin{aligned}
& \hat{c}_{1}\left(\mathbf{E}_{G}\right)=(\mathbf{a} \otimes \mathbf{a}) \cdot \mathbf{E}_{G}=0 \\
& \hat{c}_{2}\left(\mathbf{E}_{G}\right)=(\mathbf{b} \otimes \mathbf{b}) \cdot \mathbf{E}_{G}=0,
\end{aligned}
$$

while the corresponding linear constraint equations are

$$
\begin{aligned}
& \tilde{c}_{1}\left(\mathbf{E}_{G}\right)=(\mathbf{a} \otimes \mathbf{a}) \cdot \mathbf{E}=0 \\
& \tilde{c}_{2}\left(\mathbf{E}_{G}\right)=(\mathbf{b} \otimes \mathbf{b}) \cdot \mathbf{E}=0 .
\end{aligned}
$$

Note that the constraint equations (36) provide

$$
\begin{aligned}
& \frac{\partial \hat{c}_{1}}{\partial\left(\mathbf{E}_{G}\right)_{i j}}(\mathbf{O})=a_{i} a_{j} \\
& \frac{\partial \hat{c}_{2}}{\partial\left(\mathbf{E}_{G}\right)_{i j}}(\mathbf{O})=b_{i} b_{j} \\
& \frac{\partial^{2} \hat{c}_{1}}{\partial\left(\mathbf{E}_{G}\right)_{i j} \partial\left(\mathbf{E}_{G}\right)_{k l}}(\mathbf{O})=0 \\
& \frac{\partial^{2} \hat{c}_{2}}{\partial\left(\mathbf{E}_{G}\right)_{i j} \partial\left(\mathbf{E}_{G}\right)_{k l}}(\mathbf{O})=0 .
\end{aligned}
$$

Then formulas (20), (21), (22) for the three stress tensors can be easily modified when two constraints occur: the term $\left.\frac{\partial^{2} \hat{W}}{\partial \mathbf{E}_{G} \partial \mathbf{E}_{G}}(\mathbf{O})\right|_{\tilde{c}}$ becomes now

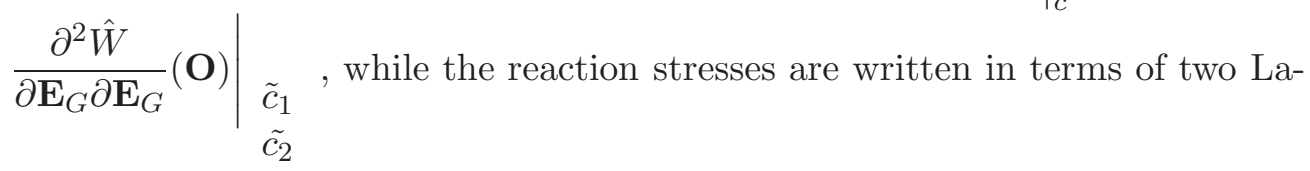
grange multipliers $q_{1}$ and $q_{2}$, so that the terms $\frac{\partial \hat{c}}{\partial \mathbf{E}_{G}}(\mathbf{O})$ and $\frac{\partial^{2} \hat{c}}{\partial \mathbf{E}_{G} \partial \mathbf{E}_{G}}(\mathbf{O})$ are now substituted by (38).

By substituting (33), (36), (37), (38) into (20), (21), (22) we obtain for the three stress tensors the following expressions

$$
\begin{array}{cl}
\mathbf{T}= & \mathbf{T}_{d}+q_{1}\left\{\mathbf{a} \otimes \mathbf{a}+\mathbf{H}(\mathbf{a} \otimes \mathbf{a})+(\mathbf{a} \otimes \mathbf{a}) \mathbf{H}^{T}\right\} \\
& +q_{2}\left\{\mathbf{b} \otimes \mathbf{b}+\mathbf{H}(\mathbf{b} \otimes \mathbf{b})+(\mathbf{b} \otimes \mathbf{b}) \mathbf{H}^{T}\right\} \\
\mathbf{S}= & \mathbf{T}_{d}+q_{1}\{\mathbf{a} \otimes \mathbf{a}+\mathbf{H}(\mathbf{a} \otimes \mathbf{a})+(\operatorname{tr} \mathbf{H}) \mathbf{a} \otimes \mathbf{a}\} \\
& +q_{2}\{\mathbf{b} \otimes \mathbf{b}+\mathbf{H}(\mathbf{b} \otimes \mathbf{b})+(\operatorname{tr} \mathbf{H}) \mathbf{b} \otimes \mathbf{b}\} \\
\tilde{\mathbf{T}}= & \mathbf{T}_{d}+q_{1}\{\mathbf{a} \otimes \mathbf{a}+(\operatorname{tr} \mathbf{H}) \mathbf{a} \otimes \mathbf{a}\} \\
& +q_{2}\{\mathbf{b} \otimes \mathbf{b}+(\operatorname{tr} \mathbf{H}) \mathbf{b} \otimes \mathbf{b}\},
\end{array}
$$


where the determinate stress $\mathbf{T}_{d}$ is given by

$$
\begin{aligned}
\mathbf{T}_{d}= & (\operatorname{tr} \mathbf{H})\left(\alpha_{11} \mathbf{I}+\alpha_{41} \mathbf{a} \otimes \mathbf{a}+\alpha_{61} \mathbf{b} \otimes \mathbf{b}\right) \\
& +\alpha_{2}\left(\mathbf{H}+\mathbf{H}^{T}\right)+\frac{1}{2} \alpha_{5}\left\{\mathbf{H}(\mathbf{a} \otimes \mathbf{a})+\mathbf{H}^{T}(\mathbf{a} \otimes \mathbf{a})\right. \\
& \left.+(\mathbf{a} \otimes \mathbf{a}) \mathbf{H}+(\mathbf{a} \otimes \mathbf{a}) \mathbf{H}^{T}\right\} \\
& +\frac{1}{2} \alpha_{7}\left\{\mathbf{H}(\mathbf{b} \otimes \mathbf{b})+\mathbf{H}^{T}(\mathbf{b} \otimes \mathbf{b})+(\mathbf{b} \otimes \mathbf{b}) \mathbf{H}\right. \\
& \left.+(\mathbf{b} \otimes \mathbf{b}) \mathbf{H}^{T}\right\} .
\end{aligned}
$$

(ii) Fibre-reinforced incompressible materials For incompressible materials equations (6), (23) become

$$
\operatorname{det}\left(2 \mathbf{E}_{G}+\mathbf{I}\right)-1=0
$$

and

$$
\operatorname{tr} \mathbf{E}=0
$$

respectively.

Equation (43) provides

$$
\begin{aligned}
& \frac{\partial \hat{c}}{\partial\left(\mathbf{E}_{G}\right)_{i j}}(\mathbf{O})=\delta_{i j} \\
& \frac{\partial^{2} \hat{c}}{\partial\left(\mathbf{E}_{G}\right)_{i j} \partial\left(\mathbf{E}_{G}\right)_{k l}}(\mathbf{O})=-2 \delta_{i k} \delta_{j l}-2 \delta_{i l} \delta_{j k}+4 \delta_{i j} \delta_{k l} .
\end{aligned}
$$

By substituting (33), (43), (44), (45) into (20), (21), (22) we obtain

$$
\begin{gathered}
\mathbf{T}=\mathbf{T}_{d}-p \mathbf{I} \\
\mathbf{S}=\mathbf{T}_{d}-p\left(\mathbf{I}-\mathbf{H}^{T}\right) \\
\tilde{\mathbf{T}}=\mathbf{T}_{d}-p\left(\mathbf{I}-\mathbf{H}-\mathbf{H}^{T}\right),
\end{gathered}
$$


where $p$ denotes the hydrostatic pressure and $\mathbf{T}_{d}$ is given by

$$
\begin{aligned}
\mathbf{T}_{d}= & \frac{1}{2} \alpha_{41}\left(\mathbf{H a} \cdot \mathbf{a}+\mathbf{H}^{T} \mathbf{a} \cdot \mathbf{a}\right) \mathbf{I} \\
& +\frac{1}{2} \alpha_{61}\left(\mathbf{H b} \cdot \mathbf{b}+\mathbf{H}^{T} \mathbf{b} \cdot \mathbf{b}\right) \mathbf{I} \\
& +\frac{1}{2} \alpha_{44}\left(\mathbf{H a} \cdot \mathbf{a}+\mathbf{H}^{T} \mathbf{a} \cdot \mathbf{a}\right) \mathbf{a} \otimes \mathbf{a} \\
& +\frac{1}{2} \alpha_{66}\left(\mathbf{H b} \cdot \mathbf{b}+\mathbf{H}^{T} \mathbf{b} \cdot \mathbf{b}\right) \mathbf{b} \otimes \mathbf{b} \\
& +\frac{1}{2} \alpha_{64}\left\{\left(\mathbf{H a} \cdot \mathbf{a}+\mathbf{H}^{T} \mathbf{a} \cdot \mathbf{a}\right) \mathbf{b} \otimes \mathbf{b}\right. \\
& \left.+\left(\mathbf{H b} \cdot \mathbf{b}+\mathbf{H}^{T} \mathbf{b} \cdot \mathbf{b}\right) \mathbf{a} \otimes \mathbf{a}\right\} \\
& +\alpha_{2}\left(\mathbf{H}+\mathbf{H}^{T}\right)+\frac{1}{2} \alpha_{5}\{\mathbf{H}(\mathbf{a} \otimes \mathbf{a}) \\
& \left.+\mathbf{H}^{T}(\mathbf{a} \otimes \mathbf{a})+(\mathbf{a} \otimes \mathbf{a}) \mathbf{H}+(\mathbf{a} \otimes \mathbf{a}) \mathbf{H}^{T}\right\} \\
& +\frac{1}{2} \alpha_{7}\left\{\mathbf{H}(\mathbf{b} \otimes \mathbf{b})+\mathbf{H}^{T}(\mathbf{b} \otimes \mathbf{b})\right. \\
& \left.+(\mathbf{b} \otimes \mathbf{b}) \mathbf{H}+(\mathbf{b} \otimes \mathbf{b}) \mathbf{H}^{T}\right\}
\end{aligned}
$$

(iii) Fibre-reinforced incompressible materials inextensible in both fibre directions In this case equations (36), (43) hold, so that the linear constraint equations are given by (37), (44).

We can now impose the linear constraint of incompressibility (44) on (39), (42), or equivalently we can impose (37) on (46), (49).

We obtain

$$
\begin{aligned}
\mathbf{T}= & \mathbf{T}_{d}-p \mathbf{I}+q_{1}\left\{\mathbf{a} \otimes \mathbf{a}+\mathbf{H}(\mathbf{a} \otimes \mathbf{a})+(\mathbf{a} \otimes \mathbf{a}) \mathbf{H}^{T}\right\} \\
& +q_{2}\left\{\mathbf{b} \otimes \mathbf{b}+\mathbf{H}(\mathbf{b} \otimes \mathbf{b})+(\mathbf{b} \otimes \mathbf{b}) \mathbf{H}^{T}\right\},
\end{aligned}
$$

where

$$
\begin{aligned}
\mathbf{T}_{d}= & \alpha_{2}\left(\mathbf{H}+\mathbf{H}^{T}\right)+\frac{1}{2} \alpha_{5}\left\{\mathbf{H}(\mathbf{a} \otimes \mathbf{a})+\mathbf{H}^{T}(\mathbf{a} \otimes \mathbf{a})\right. \\
& \left.+(\mathbf{a} \otimes \mathbf{a}) \mathbf{H}+(\mathbf{a} \otimes \mathbf{a}) \mathbf{H}^{T}\right\}+\frac{1}{2} \alpha_{7}\{\mathbf{H}(\mathbf{b} \otimes \mathbf{b}) \\
& \left.+\mathbf{H}^{T}(\mathbf{b} \otimes \mathbf{b})+(\mathbf{b} \otimes \mathbf{b}) \mathbf{H}+(\mathbf{b} \otimes \mathbf{b}) \mathbf{H}^{T}\right\} .
\end{aligned}
$$

Moreover the constitutive equations for the two Piola-Kirchhoff stresses are

$$
\begin{aligned}
& \mathbf{S}=\mathbf{T}_{d}-p\left(\mathbf{I}-\mathbf{H}^{T}\right)+q_{1}(\mathbf{I}+\mathbf{H}) \mathbf{a} \otimes \mathbf{a} \\
& +q_{2}(\mathbf{I}+\mathbf{H}) \mathbf{b} \otimes \mathbf{b} \\
& \tilde{\mathbf{T}}=\mathbf{T}_{d}-p\left(\mathbf{I}-\mathbf{H}-\mathbf{H}^{T}\right)+q_{1} \mathbf{a} \otimes \mathbf{a}+q_{2} \mathbf{b} \otimes \mathbf{b},
\end{aligned}
$$

where $\mathbf{T}_{d}$ is given by (51). 


\section{Comparison between LFTE and CLTE}

In this section we compare the constitutive equations obtained in Section 4 for LFTE with the corresponding equations usually adopted in the classical approach.

In particular we refer to equations provided by Spencer in [4], Section 6.8, and [5], Section 2.2, where the classical linear constitutive equations for constrained materials reinforced by two families of fibres are extensively discussed.

Comparison shows that the constitutive equations used in CLTE are not accurate to first order in the strain; similar results hold for other material symmetries and other constraints (see [1], [2], [3], [10], [11]).

(i) Fibre-reinforced materials inextensible in both fibre directions We compare our formulas (39), (42) with the corresponding expressions, denoted by "cl", given by Spencer in [5], formulas (39), (40).

Comparison shows that $\mathbf{T}_{d}=\mathbf{T}_{d}^{(c l)}$ while $\mathbf{T}_{r} \neq \mathbf{T}_{r}^{(c l)}$; referring to (39) we have

$$
\begin{aligned}
\mathbf{T}_{r}= & \mathbf{T}_{r}^{(c l)}+q_{1}\left\{\mathbf{H}(\mathbf{a} \otimes \mathbf{a})+(\mathbf{a} \otimes \mathbf{a}) \mathbf{H}^{T}\right\} \\
& +q_{2}\left\{\mathbf{H}(\mathbf{b} \otimes \mathbf{b})+(\mathbf{b} \otimes \mathbf{b}) \mathbf{H}^{T}\right\} .
\end{aligned}
$$

Moreover CLTE requires $\mathbf{T}^{(c l)}=\mathbf{S}^{(c l)}=\tilde{\mathbf{T}}^{(c l)}$, so that (40), (41), (54) provide

$$
\begin{aligned}
\mathbf{S}_{d}= & \mathbf{S}_{d}^{(c l)} \\
\tilde{\mathbf{T}}_{d}= & \tilde{\mathbf{T}}_{d}^{(c l)} \\
\mathbf{S}_{r}= & \mathbf{S}_{r}^{(c l)}+q_{1}\{\mathbf{H}(\mathbf{a} \otimes \mathbf{a})+(\operatorname{tr} \mathbf{H}) \mathbf{a} \otimes \mathbf{a}\}+ \\
& +q_{2}\{\mathbf{H}(\mathbf{b} \otimes \mathbf{b})+(\operatorname{tr} \mathbf{H}) \mathbf{b} \otimes \mathbf{b}\} \\
\tilde{\mathbf{T}}_{r}= & \tilde{\mathbf{T}}_{r}^{(c l)}+q_{1}(\operatorname{tr} \mathbf{H}) \mathbf{a} \otimes \mathbf{a}+q_{2}(\operatorname{tr} \mathbf{H}) \mathbf{b} \otimes \mathbf{b} .
\end{aligned}
$$

Therefore the three stress tensors used in CLTE are not correct.

(ii) Fibre-reinforced incompressible materials We now compare (46), (49) with the corresponding expressions given by Spencer in [5], pp. 12-13. We see that $\mathbf{T}_{r}=\mathbf{T}_{r}^{(c l)}$, while $\mathbf{T}_{d} \neq \mathbf{T}_{d}^{(c l)}$; referring to (49) we have

$$
\begin{aligned}
\mathbf{T}_{d}= & \mathbf{T}_{d}^{(c l)}+\frac{1}{2} \alpha_{41}\left(\mathbf{H a} \cdot \mathbf{a}+\mathbf{H}^{T} \mathbf{a} \cdot \mathbf{a}\right) \mathbf{I} \\
& +\frac{1}{2} \alpha_{61}\left(\mathbf{H b} \cdot \mathbf{b}+\mathbf{H}^{T} \mathbf{b} \cdot \mathbf{b}\right) \mathbf{I}
\end{aligned}
$$


Moreover (47), (48), (56) provide

$$
\begin{aligned}
\mathbf{S}_{d}= & \mathbf{S}_{d}^{(c l)}+\frac{1}{2} \alpha_{41}\left(\mathbf{H a} \cdot \mathbf{a}+\mathbf{H}^{T} \mathbf{a} \cdot \mathbf{a}\right) \mathbf{I} \\
& +\frac{1}{2} \alpha_{61}\left(\mathbf{H b} \cdot \mathbf{b}+\mathbf{H}^{T} \mathbf{b} \cdot \mathbf{b}\right) \mathbf{I} \\
\tilde{\mathbf{T}}_{d}= & \tilde{\mathbf{T}}_{d}^{(c l)}+\frac{1}{2} \alpha_{41}\left(\mathbf{H a} \cdot \mathbf{a}+\mathbf{H}^{T} \mathbf{a} \cdot \mathbf{a}\right) \mathbf{I} \\
& +\frac{1}{2} \alpha_{61}\left(\mathbf{H b} \cdot \mathbf{b}+\mathbf{H}^{T} \mathbf{b} \cdot \mathbf{b}\right) \mathbf{I} \\
\mathbf{S}_{r}= & \mathbf{S}_{r}^{(c l)}+p \mathbf{H}^{T} \\
\tilde{\mathbf{T}}_{r}= & \tilde{\mathbf{T}}_{r}^{(c l)}+p\left(\mathbf{H}+\mathbf{H}^{T}\right) .
\end{aligned}
$$

Also in this case the three stress tensors $\mathbf{T}^{(c l)}, \mathbf{S}^{(c l)}, \tilde{\mathbf{T}}^{(c l)}$ are not correct.

(iii) Fibre-reinforced incompressible materials inextensible in both fibre directions We compare formulas (50), (51) with the constitutive equations provided by Spencer in [5], formulas (41), (42).

The determinate part of $\mathbf{T}^{(c l)}$ profided by CLTE is correct $\left(\mathbf{T}_{d}=\mathbf{T}_{d}^{(c l)}\right)$, while the reaction part is not correct; in fact referring to (50) we can see that

$$
\begin{aligned}
\mathbf{T}_{r}= & \mathbf{T}_{r}^{(c l)}+q_{1}\left\{\mathbf{H}(\mathbf{a} \otimes \mathbf{a})+(\mathbf{a} \otimes \mathbf{a}) \mathbf{H}^{T}\right\} \\
& +q_{2}\left\{\mathbf{H}(\mathbf{b} \otimes \mathbf{b})+(\mathbf{b} \otimes \mathbf{b}) \mathbf{H}^{T}\right\} .
\end{aligned}
$$

Moreover (52), (53), (58) provide

$$
\begin{aligned}
& \mathbf{S}_{d}=\mathbf{S}_{d}^{(c l)} \\
& \tilde{\mathbf{T}}_{d}=\tilde{\mathbf{T}}_{d}^{(c l)} \\
& \mathbf{S}_{r}=\mathbf{S}_{r}^{(c l)}+p \mathbf{H}^{T}+q_{1} \mathbf{H}(\mathbf{a} \otimes \mathbf{a})+q_{2} \mathbf{H}(\mathbf{b} \otimes \mathbf{b}) \\
& \tilde{\mathbf{T}}_{r}=\tilde{\mathbf{T}}_{r}^{(c l)}+p\left(\mathbf{H}+\mathbf{H}^{T}\right) .
\end{aligned}
$$

As in case (i) only the determinate part of the three stress tensors used in CLTE is correct.

\section{Conclusions}

This paper represents a natural carrying on of [1], [2], [6], [7], [8], [9], [10], [11], since the linearized finite theory of elasticity is here applied to constrained materials with orthotropic symmetry.

In this paper by using the linearized finite theory of elasticity we obtain the constitutive equations for constrained hyperelastic materials reinforced by 
two orthogonal families of fibres. In particular we derive stress-strain relations for materials subject to the constraint of inextensibility in both fibre directions and for materials subject to the constraint of incompressibility.

The constitutive equations obtained in this paper significantly differ from those of the classical linear elasticity. The same occurs for other material symmetries and other constraints, since only the linearized finite theory of elasticity exhibits the accuracy required by a linear model.

\section{Acknowledgments}

This research was supported by the Department of Mathematics "Giuseppe Peano" of the University of Turin (Italy).

\section{References}

[1] A. Hoger, B.E. Johnson, Linear elasticity for constrained materials: Incompressibility, J. Elasticity, 38 (1995), 69-93, doi: 10.1007/BF00121464.

[2] A. Hoger, B.E. Johnson, Linear elasticity for constrained materials: General theory for hyperelasticity, J. Elasticity, 38 (1995), 95-120, doi: 10.1007/BF00121465.

[3] R.S. Marlow, On the stress in an internally constrained elastic material, J. Elasticity, 27 (1992), 97-131, doi: 10.1007/BF00041645.

[4] A.J.M. Spencer, Deformations of Fibre-Reinforced Materials, Oxford University Press, Oxford (1972).

[5] A.J.M. Spencer, Continuum theory of the mechanics of fibre-reinforced composites, Springer Verlag, Wien (1984), doi: 10.1007/978-3-7091-4336-0.

[6] M.L. Tonon, Waves in constrained linear elastic materials, J. Elasticity, 69 (2002), 15-39, doi: 10.1023/A:1027334213793.

[7] M.L. Tonon, Wave propagation according to the linearized finite theory of elasticity, International J. of Pure and Applied Mathematics, 40, No. 1 (2007), 135-152.

[8] M.L. Tonon, A note on the linearized finite theory of elasticity, International J. of Pure and Applied Mathematics, 58, No. 2 (2010), 195-208.

[9] M.L. Tonon, On simple shear for incompressible isotropic linear elastic materials, International J. of Pure and Applied Mathematics, 75, No. 3 (2012), 353-370.

[10] M.L. Tonon, On the three stress tensors for linearly elastic constrained materials, International J. of Pure and Applied Mathematics, 91, No. 4 (2014), 459-475, doi: 10.12732/ijpam.v91i4.3.

[11] M.L. Tonon, Second-order stress relations for hyperelastic constrained materials, International J. of Pure and Applied Mathematics, 93, No. 6 (2014), 813-833, doi: 10.12732/ijpam.v93i6.8. 